$7^{\text {th }}$ Jagna International Workshop (2014)

International Journal of Modern Physics: Conference Series

Vol. 36 (2015) 1560009 (6 pages)

(C) The Author

DOI: 10.1142/S2010194515600095

\title{
Bier-Astumian relation, fluctuation theorem and their possible applications
}

\author{
Mark Nolan P. Confesor \\ Department of Physics, MSU-Iligan Institute of Technology, \\ Iligan City, 9200, Philippines \\ marknolan2006@gmail.com
}

Published 2 January 2015

\begin{abstract}
Fluctuations in the spatial position of a probe particle that is driven far from equilibrium can provide valuable information about the driving force. Analysis of the position fluctuation is through the fluctuation theorem (FT) and a generalized detailed balance called Bier-Astumian relation (BA). Here we show the usefulness of the BA for mapping potential landscapes of a particle confined in a potential field. We also demonstrate how the FT can be used to extract the driving force for a particle driven by a constant force.
\end{abstract}

Keywords: Detailed balance condition; fluctuation theorem.

\section{Introduction}

There have been great strides in fully developing nanotechnology due to possible uses in medicine, computation and advanced materials among others. Interestingly, such envisioned application of nanotechnology presents fundamental questions in physics such as in the understanding of the effect of thermal noise on the operation of sub- $\mu m$ sized motors in statistical mechanics [1].

For small systems, the presence of thermal noise naturally leads to fluctuations in some observable quantities conduit to the operation of a particular bio/chem/mechanical operation [2]. One case is when thermal noise leads to the fluctuation in position of a kinesin molecular motor moving in a microtubule [3]. The approach of extracting useful information on the thermodynamics of a system from fluctuating observables has become experimentally practical through the use of $\mu m$-sized colloidal beads as probe particles attached to the system of interest, such as beads attached to a $\mathrm{F}_{1}$-ATPase [4], optically trapped beads dragged in water [5] and an optically trapped bead under a temperature gradient $[6,7]$.

This is an Open Access article published by World Scientific Publishing Company. It is distributed under the terms of the Creative Commons Attribution 3.0 (CC-BY) License. Further distribution of this work is permitted, provided the original work is properly cited. 
In this article we will present two methods that can be used to analyze the fluctuating position of the probe particle. Our approach is not to provide rigorous mathematical treatment of the methods but rather we will focus more on how such methods can be used to extract thermodynamic variables in the system at hand.

\section{Bier-Astumian Relation}

For systems in thermodynamic equilibrium, the Detailed Balance (DB) leads to the Boltzmann distribution being the stationary solution of the Master's equation. The $D B$ is basically a measure of reversibility,

$$
\frac{P\left(x_{a} \rightarrow x_{b} ; \Delta t\right)}{P\left(x_{b} \rightarrow x_{a} ; \Delta t\right)}=\frac{P_{e q}\left(x_{b}\right)}{P_{e q}\left(x_{a}\right)}
$$

where $P\left(x_{i} \rightarrow x_{j}\right)$ is the transition probability to move from position $x_{i}$ to $x_{j}$ and $P_{e q}(x)$ is the position probability distribution [8]. We note that LHS of Eq. 1 is a ratio of two time dependent functions while on the RHS this time dependence vanishes. In Ref. [9], the DB was generalized (the Bier-Astumian relation) to cases when particles are moving in medium with a spatial gradient of a thermodynamic variable $F(x)$, i.e. phoretic transport. For instance in diffusiophoresis particles move due to the presence of solute concentration gradients and in electrophoresis when there is an electric potential gradient [10]. The Bier-Astumian relation (BA) has the form,

$$
\frac{P\left(x_{a} \rightarrow x_{b} ; \Delta t\right)}{P\left(x_{b} \rightarrow x_{a} ; \Delta t\right)}=e^{-\frac{\Delta G}{k_{B} T}}
$$

where $\Delta G=G\left(F\left(x_{b}\right)\right)-G\left(F\left(x_{a}\right)\right)$ and $G(F(x))$ is the free energy. $k_{B} T$ is the Boltzman factor. In thermophoresis, the free energy difference is updated to include the kinetic energy difference between $x_{a}$ and $x_{b}[6]$.

We performed brownian dynamic simulation of a probe particle trapped in a harmonic well to check the validity of the BA, schematics of the set-up is shown in Figure 1.a. The probe particle dynamics is described by the Langevin equation of the form [11],

$$
\gamma \frac{d x}{d t}=-k x+\xi(t)
$$

where $\gamma=6 \pi \eta a(0.017 \mathrm{pN} \cdot \mathrm{s} / \mu m), k(1.37 \mathrm{pN} / \mu \mathrm{m})$ is the trap stiffness and $\xi(t)$ is a white noise with $\langle\xi(t)\rangle=0$ and $\left\langle\xi(t) \xi\left(t^{\prime}\right)\right\rangle=2 \gamma k_{B} T \delta\left(t-t^{\prime}\right)\left(k_{B} T=0.004 \mathrm{pN} \cdot \mu \mathrm{m}\right)$. Probe position is computed via the time difference form of Eq. 3. Each simulation generated trajectories consisting of $10^{4}$ particle positions in intervals of $\Delta t=1 \mathrm{~ms}$. In the inset of Figure 2.a we plot the probe position probability distribution which is well fitted by a normal distribution as expected for a harmonic trap. As a means of checking, the trap stiffness value was recovered from the standard deviation, $\left\langle\Delta x^{2}\right\rangle$, via the relation, $k=\frac{k_{B} T}{\left\langle\Delta x^{2}\right\rangle}$ and from the expected form of the autocorrelation function, $\langle x(t+\Delta t) x(t)\rangle \sim \exp \left[-\frac{t}{\tau}\right]$, where $k=\frac{\gamma}{\tau}[11]$. In Figure 2.b we plot the 


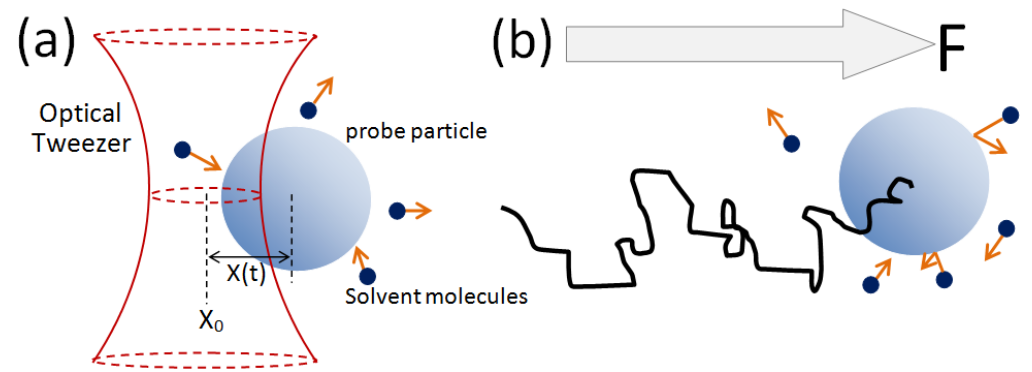

Fig. 1. (a) Probe particle trapped in an optical tweezer. Thermal fluctuations causes the probe particle to deviate from the trap center $\left(X_{0}\right)$. (b) Illustration of a typical trajectory of a probe particle under an external force contains a stochastic and a net drift component.

transition probabilities for the probe particle to jump from $x=0$ (the trap center) to a position $x, P(0 \rightarrow x ; \Delta t)$, and the inverse transition probabilities, $P(x \rightarrow 0 ; \Delta t)$. As expected both transition probabilities show different profiles for different time intervals where the profile spread more for $\Delta t=10 \mathrm{~ms}$ than for $\Delta t=2 \mathrm{~ms}$. From the Smoluchowski equation for a particle trapped in a harmonic well, the transition probabilities are computed and take the form [11],

$$
P\left(x \rightarrow x^{\prime} ; \Delta t\right)=F(\Delta t) \exp \left[\frac{k\left[x-x^{\prime} \exp \left(-\frac{\Delta t}{\tau}\right)\right]^{2}}{2 k_{B} T\left[1-\exp \left(-\frac{2 \Delta t}{\tau}\right)\right]}\right],
$$

where $F(\Delta t)$ is a prefactor. As observed, the spreading of $P\left(x \rightarrow x^{\prime} ; \Delta t\right)$ increases for bigger $\Delta t$. Furthermore, one can then easily show that from the transition probabilities, Eq. 4, the BA holds,

$$
\frac{P\left(x_{a} \rightarrow x_{b} ; \Delta t\right)}{P\left(x_{b} \rightarrow x_{a} ; \Delta t\right)}=\exp \left[\frac{k}{2} \frac{x_{a}{ }^{2}-x_{b}{ }^{2}}{k_{B} T}\right]=\exp \left[-\frac{\Delta G}{k_{B} T}\right]
$$

Simulation results also provide validity of the BA as seen by the recovery of the trapping potential well from the ratio of the transition probabilities, $\frac{\Delta U(x)}{k_{B} T}=$ $\log \left(\frac{P(x \rightarrow 0 ; \Delta t)}{P(0 \rightarrow x ; \Delta t)}\right)$. The computed potential wells were found to be in excellent agreement with that obtained by the traditional method of inverting the Boltzmann distribution, i.e. $U(x)=-k_{B} T \log P_{e q}(x)$ as shown in Figure 2.c.

\section{Fluctuation Theorem}

Generally the fluctuation theorem quantifies the asymmetry in the entropy production of a given system. It is written as,

$$
\lim _{\Delta t \rightarrow \infty} \frac{k_{B}}{\Delta t} \ln \left[\frac{P_{\Delta t}(\sigma)}{P_{\Delta t}(-\sigma)}\right]=\sigma
$$

where $\sigma$ is the entropy production and $P_{\Delta t}(\sigma)$ is the time dependent probability distribution for the entropy production [3]. For a colloidal bead immersed in a medium (equilibrated with temperature $T$ ) and under an external force, $F$, the 

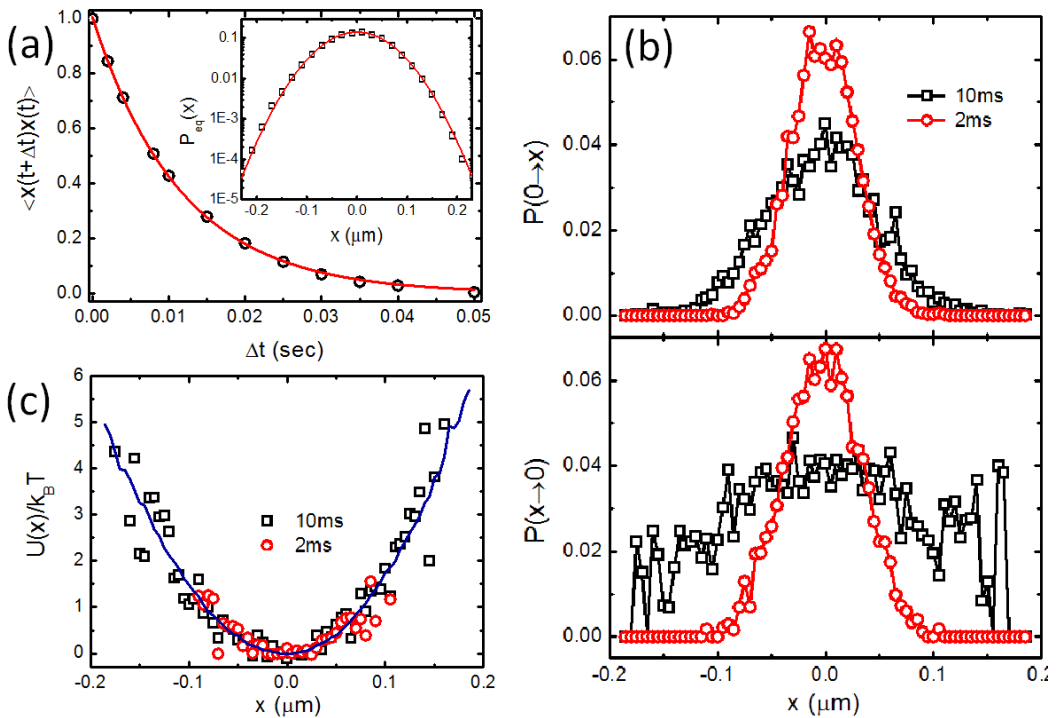

Fig. 2. (a) Autocorrelation function of $x(t)$ for different lag times. Solid line is an exponential fit, $\langle x(t+\Delta t) x(t)\rangle=\exp [-\Delta t / \tau]$ where $\tau=0.012 \mathrm{~s}$. Inset shows the probability distribution of the particle position. Fitting curve is a normal distribution fitting with $\langle x\rangle=0 \mu m$ and $\left\langle\Delta x^{2}\right\rangle=$ $3 \times 10^{-3} \mu m^{2}$. (b) Transition probabilities computed for transition from the trap center $(x=0 \mu \mathrm{m})$ to a position $x$ and vice versa. (c) Trapping potential as recovered by using the BA (open symbols) and through inverting the Boltzman distribution (solid line).

entropy production is just the rate of heat exchange $(Q)$ of the particle to the bath at some time interval $\Delta t$, i.e. $\sigma=Q / T \Delta t$. Furthermore, the heat exchange is given by $Q=\vec{F} \cdot \overrightarrow{\Delta x}$ (1-dim), where $\Delta x$ is the particle incremental step. For the case when $F=0 \mathrm{pN}$, the ratio of $P_{\Delta t}(\sigma) / P_{\Delta t}(-\sigma)$ equals 1 since there is equal probability for the particle to take $+\Delta x$ and $-\Delta x$, i.e. only when there is driving that the ratio is different from 1 . In this section we will show the usefulness of the FT in extracting the driving force of a probe particle subjected to a constant external forcing, schematics in Figure 1.b. We performed brownian dynamic simulation of a Langevin equation of the form,

$$
\gamma \frac{d x}{d t}=F+\xi(t)
$$

where we varied $F$ from $0.0 \mathrm{pN} \rightarrow 0.04 \mathrm{pN}$. The distribution of $\sigma$ is plotted in the inset of Figure 3.a for two different $\Delta t$ for the case when $F=0.01 \mathrm{pN}$. We have observed that at longer $\Delta t$ the asymmetry of the distribution becomes more pronounced. Furthermore, we also verified the validity of the FT for different $\Delta t$ as shown in Figure 3.a. The practical use of the FT is elucidated in Figure 3.b, where we generate particle trajectories corresponding to different $F$. We then construct the corresponding probability distribution of $\Delta x$ for $\Delta t=0.30 \mathrm{~s}$ and computed the ratio for forward and backward steps as a function of $\Delta x$. We see a linear relation between $\ln \left[P_{\Delta t}(\Delta x) / P_{\Delta t}(-\Delta x)\right]$ and $\Delta x$ such that the y-intercept is the zero point 

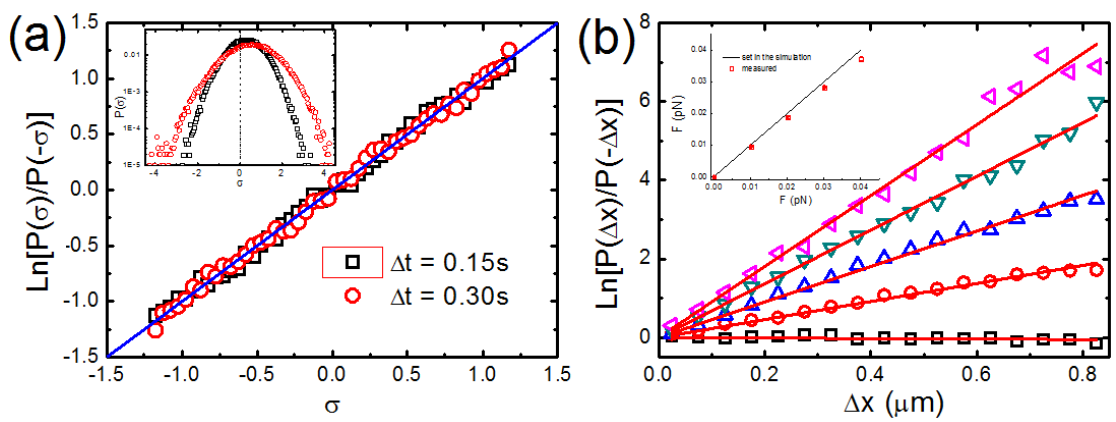

Fig. 3. (a) Verification of the FT, Eq. 6, for the case when $F=0.01 \mathrm{pN}$. Inset shows the distribution of $\sigma$ for $\Delta t=0.3 \mathrm{~s}$ and $\Delta t=0.15 \mathrm{~s}$ (b) Ratio of the forward and backward steps distribution of particle position for different values of $F$; $(\square) 0.0 \mathrm{pN},(\bigcirc) 0.01 \mathrm{pN},(\triangle) 0.02 \mathrm{pN}$, $(\nabla) 0.03 \mathrm{pN}$ and $(\triangleleft) 0.04 \mathrm{pN}$. Solid line is a linear fitting with intercept equal to zero. Inset shows the computed force (symbols) to the set values in the simulation (solid line).

as is predicted in Eq. 6. Since $\ln \left[P_{\Delta t}(\Delta x) / P_{\Delta t}(-\Delta x)\right]=\ln \left[P_{\Delta t}(\sigma) / P_{\Delta t}(-\sigma)\right]$ and $\sigma=\frac{F \Delta x}{T \Delta t}$ then the slope of the lines corresponds to $\frac{F}{k_{B} T}$, i.e. $F=$ slope $/ k_{B} T$. In the inset of Figure 3.b we plot the computed force from the slope and compared it to the set value of $F$ in the simulation and found good agreements. Lastly, we note that the method described in this section in using FT to measure $F$ was already applied to real systems whose dynamics is described by a Langevin equation similar to Eq. 7, such as the estimation of torque in the rotation of self-propelling dimers [12] and the rotation of $\mathrm{F}_{1}$-ATPase motor [4].

\section{Conclusion}

The usefulness of the BA and the FT in extracting thermodynamic variables have been illustrated via simulation for two simple systems. Using the BA the trapping potential was recovered for the case of a probe particle in a harmonic trap. We expect the BA to be able to map even more complicated potential landscapes [13]. Furthermore, the FT was successfully used to recover the driving force for the case of a probe particle under the action of a constant force. The applicability of FT will allow it to measure non-equilibrium driving forces for instance thermophoretic force in thermophoresis [7]. The FT was also used to extract the torque that causes an asymmetric wheel to rotate in the presence of self-propelling particles in the granular scale [14].

\section{Acknowledgments}

Insightful discussions with Prof. C. K. Chan and Prof. Pik-Yin Lai are greatly acknowledged. 


\section{References}

1. R. Phillips and S. R. Quake, Phys. Today 59(5), 38 (2006).

2. R. Dean Astumian, Science 276, 917 (1997).

3. C. Bustamante et. al, Phys. Today 58(7), 43 (2005).

4. K. Hayashi et. al, Phys. Rev. Lett. 104, 218103 (2010).

5. D. Andrieux et. al, Phys. Rev. Lett. 98, 150601 (2007).

6. M. N. Confesor and P. Y. Lai, Chin. J. Phys. 51, 522 (2013).

7. M. N. Confesor, P. Y. Lai and C. K. Chan in preparation.

8. F. Ritort, Adv. Chem. Phys. 137, (2008).

9. R. Dean Astumian and R. Brody, J. Phys. Chem. B 113, 11459 (2009).

10. J. L. Anderson, Ann. Rev. of Fluid Mech. 21, 61-69 (1989).

11. M. Doi and S. F. Edwards, The Theory of Polymer Dynamics, (Clarendon Press, 1998).

12. R. Suzuki, H. R. Jiang and M. Sano, arXiv:1104.560\%.

13. X. G. Ma, P. Y. Lai and P. Tong, Soft Matter 9, 8826 (2013).

14. E. Hamoy and M. N. Confesor, to be submitted. 